\title{
Factors associated with success and failure of patient-controlled oral analgesia after total hip and knee arthroplasty: a historical comparative cohort study
}

\section{Facteurs associés à la réussite ou à l'échec de l'analgésie orale contrôlée par le patient après une arthroplastie totale de la hanche ou du genou : une étude de cohorte historique comparative}

\author{
Leon Vorobeichik, MD, FRCPC · Yasmine Hoydonckx, MD, FIPP · Pranab Kumar, MD, \\ FRCPC, FRCA, FFPMRCA • Arlene Buzon-Tan, RN(EC) - Susan Walker, RN(EC) • \\ Kyle Kirkham, MD, FRCPC • Dharini Ilangomaran, BSc $\cdot$ Lashmi Venkatraghavan, MD, \\ FRCPC, FRCA · Atul J. Prabhu, MD, FRCA, FRCPC · Anuj Bhatia, MD, FRCPC, \\ FRCA, FFPMRCA iD
}

Received: 12 January 2020/Revised: 25 August 2020/Accepted: 31 August 2020/Published online: 17 November 2020

(C) Canadian Anesthesiologists' Society 2020

\begin{abstract}
Purpose Patient-controlled oral analgesia (PCOA) is a novel method of oral opioid administration using set doses of short-acting oral opioids self-administered by patients with a "lockout" period as part of a multimodal regimen. Failure of PCOA can result in severe postoperative pain necessitating use of intravenous patient-controlled analgesia (IV-PCA) with its potential complications. This study evaluated factors related to success or failure of
\end{abstract}

Electronic supplementary material The online version of this article (https://doi.org/10.1007/s12630-020-01864-5) contains supplementary material, which is available to authorized users.

L. Vorobeichik, MD, FRCPC

Department of Anesthesia and Pain Medicine, Faculty of

Medicine, University of Toronto, Toronto, ON, Canada

Y. Hoydonckx, MD, FIPP · P. Kumar, MD, FRCPC, FRCA, FFPMRCA $\cdot$ K. Kirkham, MD, FRCPC $\cdot$ L. Venkatraghavan, MD, FRCPC, FRCA · A. J. Prabhu, MD, FRCA, FRCPC Department of Anesthesia and Pain Medicine, Faculty of Medicine, University of Toronto, Toronto, ON, Canada

Department of Anesthesia and Pain Management, Toronto Western Hospital - University Health Network, Toronto, ON, Canada

A. Buzon-Tan, RN(EC) - S. Walker, RN(EC) .

D. Ilangomaran, BSc

Department of Anesthesia and Pain Management, Toronto

Western Hospital - University Health Network, Toronto, ON,

Canada
PCOA following total hip arthroplasty (THA) and total knee arthroplasty (TKA).

Methods We conducted a retrospective cohort study of all adults who underwent THA and TKA at our institution by extracting data from the proprietary database of our acute pain service. Patient, anesthetic, and surgical variables associated with PCOA failure defined as inadequate analgesia requiring conversion to IV-PCA within $24 \mathrm{hr}$ following THA and TKA were evaluated. Univariable and multivariable logistic regression analyses were performed to identify predictors of PCOA failure.

A. Bhatia, MD, FRCPC, FRCA, FFPMRCA ( $\square)$ Department of Anesthesia and Pain Medicine, Faculty of Medicine, University of Toronto, Toronto, ON, Canada e-mail: anuj.bhatia@uhn.ca

Department of Anesthesia and Pain Management, Toronto Western Hospital - University Health Network, Toronto, ON, Canada

Toronto Western Hospital, McL 2-405, Department of Anesthesia and Pain Management, Toronto Western Hospital, University Health Network, 399 Bathurst Street, Toronto, ON M5T 2S8, Canada 
Results Of the 926 patients who underwent THA or TKA ( $n=411$ and 515, respectively), 147 (15.9\%) patients (67 THA and 80 TKA patients) had PCOA failure with moderate-to-severe pain. Multivariable regression analysis showed that PCOA failure occurred in those with younger age (adjusted odds ratio [aOR] per year of age, 0.97; 99\% CI, 0.95 to 0.99; $P<0.001$ ), preoperative chronic use of controlled-release opioids (aOR, 3.45; $99 \%$ $C I, 1.60$ to $7.35 ; P<0.001$ ), and with the use of general anesthesia vs spinal anesthesia (aOR, 2.86; 99\% CI, 1.20 to $6.84 ; P=0.002$ ).

Conclusion The use of PCOA provides adequate analgesia to a majority of patients undergoing THA and TKA. Factors predictive for PCOA failure should be considered when choosing the primary breakthrough analgesic modality following THA/TKA.

\section{Résumé}

Objectif L'analgésie orale contrôlée par le patient $($ AOCP) est une méthode novatrice d'administration d'opiö̈les oraux qui utilise des doses pré-établies d'opioïles oraux à courte action auto-administrées par les patients avec un intervalle minimal entre les doses dans le cadre d'un régime multimodal. Le non-fonctionnement d'une AOCP peut entrainer une douleur postopératoire grave nécessitant le recours à une analgésie intraveineuse contrôlée par le patient (ACP-IV), ce qui s'accompagne de complications potentielles. Cette étude a évalué les facteurs liés à la réussite ou à l'échec de l'AOCP à la suite d'une arthroplastie totale de la hanche (ATH) ou du genou (ATG).

Méthode Nous avons réalisé une étude de cohorte rétrospective de tous les adultes ayant subi une ATH ou une ATG dans notre établissement en extrayant les données de la base de données de notre service de douleur aiguë. Les variables liées au patient, à l'anesthésie, et à la chirurgie et associées à un échec de l'AOCP, défini comme une analgésie inadéquate exigeant la conversion en ACPIV dans les 24 heures suivant l'ATH ou l'ATG, ont été évaluées. Des analyses de régression logistique univariée et multivariée ont été effectuées pour identifier les prédicteurs d'un échec de l'AOCP.

Résultats Sur les 926 patients ayant subi une ATH ou une $A T G$ ( $n=411$ et 515, respectivement), l'AOCP n'a pas fonctionné chez 147 (15,9\%) patients (67 patients d'ATH et 80 d'ATG), entrainant une douleur modérée à grave. L'analyse de régression multivariée a montré que les échecs de l'AOCP sont survenus chez les personnes plus jeunes (rapport de cotes ajusté [RCA] par année d'âge, 0,97; IC $99 \%, 0,95$ à 0,99; $P<0,001$ ), lors d'une utilisation préopératoire chronique d'opioüdes à libération contrôlée (RCA, 3,45; IC $99 \%, 1,60$ à 7,35; P<0,001), et lors d'une anesthésie générale vs une rachianesthésie (RCA, 2,86; IC $99 \%, 1,20$ à 6,84; $P=0,002$ ).

Conclusion L'utilisation de l'AOCP procure une analgésie adéquate à la majorité des patients subissant une ATH ou une ATG. Les facteurs prédictifs d'un échec de l'AOCP devraient être pris en considération lors du choix de la principale modalité analgésique après une ATH/ATG.

Keywords patient-controlled oral analgesia - PCOA . hip and knee arthroplasty

Total hip and knee arthroplasties (THA and TKA) are major orthopedic procedures that are commonly and increasingly ${ }^{1}$ performed in patients with degenerative disease of the respective joints. These operations can relieve disabling joint pain, restore gait, and improve quality of life, ${ }^{2,3}$ but are associated with moderate-tosevere early postoperative pain in over one third of patients. ${ }^{4}$ Ensuring adequate analgesia in the early postoperative period following THA and TKA is crucial because high levels of pain are associated with delayed mobilization, ${ }^{5}$ prolonged length of stay in the hospital, ${ }^{5,6}$ poor quality of sleep, ${ }^{7}$ and an increase in occurrence of persistent postoperative pain. ${ }^{8}$

Current postoperative pain management practices for joint arthroplasty in the lower limb involve multimodal therapies and display significant inter- and intrainstitutional variability. ${ }^{9}$ Parenteral administration of opioids in the form of intravenous patient-controlled analgesia (IV-PCA), based on self-regulation of opioid administration within limits defined by the anesthesiologyled acute pain service (APS), is one common method of breakthrough pain management following joint arthroplasty, ${ }^{9}$ but it requires trained staff, intravenous access, specialized equipment, ${ }^{10}$ and is associated with significant costs. ${ }^{11,12}$ Furthermore, IV-PCA may also be associated with adverse events including opioid overdose, device malfunction, and operator errors. The adverse effects of IV-PCA include nausea (incidence: 30\%), vomiting $(9 \%)$, urinary retention $(46 \%)$, and respiratory insufficiency (5\%). ${ }^{9,13}$ A study published in 2009 reported on Food and Drug Administration's Manufacturer and User Facility Device Experience data associated with epidural or IV-PCA use in the postoperative period; $6.5 \%$ of IV-PCArelated events were due to operator error. Most $(81 \%)$ of these errors were due to pump mis-programming, of which almost half were associated with patient harm; $76.4 \%$ of adverse events were attributed to device malfunction. ${ }^{14}$

There is evidence that oral opioid alternatives to IVPCA have equivalent analgesic effects in the postoperative period with a lower incidence of adverse effects. ${ }^{15-17}$ 
Patient-controlled oral analgesia (PCOA) is a novel method of oral opioid administration practiced at our institution whereby breakthrough pain is managed with a set dose of a short-acting oral opioid that is available at the patient's bedside and is replaced if exhausted following regular lockout intervals (every two hours) while patients record pain levels and opioid use. ${ }^{18}$ Patient-controlled oral analgesia may offer benefits such as a better analgesic profile, increased mobility, and less constipation, ${ }^{19}$ and allows patients to vary their analgesia more easily according to pain and activity levels, ${ }^{20}$ while minimizing costs. Evidence for PCOA is limited but supportive; the concept was first introduced in case studies. $^{21,22}$ Subsequently, a randomized-controlled trial on 60 patients who had orthopedic surgery reported that morphine PCOA was associated with analgesic benefit that was adequate and similar to that obtained with morphine IV-PCA. Furthermore, patients reported a high level of satisfaction with both PCOA and IV-PCA. ${ }^{23}$ Nevertheless, there are currently no published success rates of PCOA nor factors predictive for PCOA failure in large cohorts of patients. Such data would help perioperative physicians to identify and focus care to patients at higher risk of inadequate pain relief by guiding the selection of appropriate analgesic modalities and minimizing complications associated with inadequate pain relief in the first 24 postoperative hours. ${ }^{24}$

The objectives of this study were to establish the success and failure rates of PCOA and to evaluate patient-related, anesthetic-related, and surgery-related variables associated with failure of PCOA in the first 24 postoperative hours (as defined by moderate-to-severe pain requiring conversion to IV-PCA) in patients who underwent THA or TKA.

\section{Methods}

This manuscript is reported as per the Strengthening the Reporting of Observational Studies in Epidemiology ${ }^{25}$ and the Reporting of studies Conducted using Observational Routinely-collected health Data guidelines. ${ }^{26}$

\section{Settings and data collection}

After receiving Research Ethics Board approval (University Health Network, Toronto, Ontario, Canada; REB \# 16-6240) and waiver for consent from patients because of the anonymized nature of the data, we conducted a retrospective study at Toronto Western Hospital (a part of the University Health Network, Toronto), a high volume total hip and knee arthroplasty tertiary care centre promoting fast-track surgery with a goal of discharge by the first to second postoperative day. The protocol was not registered on a national or international registry for ongoing studies. The study extracted data from the Networked Online Processing of Acute Pain Information (NOPAIn) database developed in-house at our hospital and used to capture data on domains related to acute postoperative pain in the first few days following surgery while the patients are followed by the APS. In the NOPAIn database, perioperative data are entered by the anesthesiologist delivering intraoperative care, with data collected in the postoperative period at least twice daily by the APS team.

Data on the following variables is entered on an online portal when patients are registered in the NOPAIn database: age, sex, surgical procedure type (THA or TKA; unilateral or bilateral arthroplasty; primary or revision), comorbidities, preoperative medications by class, baseline laboratory values for serum hemoglobin and creatinine, administration of intrathecal morphine during spinal anesthesia, anesthesia technique (general or spinal anesthesia), and use of adductor canal block (ACB) for patients undergoing TKA. Data are collected on the following variables in the postoperative period in real-time during APS rounds: prescribed postoperative analgesic medications and their dosages (cumulative oral and intravenous breakthrough opioid consumption-reported as total opioid consumption in oral morphine equivalents in milligrams ${ }^{27}$ in the first 24 postoperative hours), moderateto-severe pain requiring initiation of IV-PCA (and termination of PCOA), pain severity scores at rest and with movement on an 11-point (0-10) numerical rating scale (NRS), and duration of treatment (in days) by the APS. The database is housed in a secure environment that is accessible on our hospital intranet only by hospital staff with retained digital footprints for data entry and access. Data are downloaded and backed-up at weekly intervals on a hard drive kept in a locked location. We perform quality checks on our database on a regular basis.

\section{Perioperative anesthetic and analgesic protocols}

The study analyzed all consecutive episodes of perioperative care for patients who underwent THA and TKA between 1 April 2015 (to coincide with the launch of the current version of NOPAIn) and 30 September 2016 (to coincide with substantial changes in the platform of the database resulting in a new version) and were prescribed PCOA as the primary analgesic modality. Unless contraindicated and with patient consent, THA and TKA at our hospital are performed under subarachnoid block with isobaric bupivacaine $(0.5 \%)$ administered in a dose of $12.5 \mathrm{mg}(2.5 \mathrm{~mL})$. Intrathecal morphine $(100 \mu \mathrm{g})$ is also administered in patients who do not have risk factors for respiratory depression (e.g., severe obstructive sleep apnea) 
or allergy to this medication. A single-injection ACB with $20 \mathrm{~mL}$ of $0.5 \%$ ropivacaine is performed for patients undergoing TKA. Patients undergoing TKA or THA also receive an intraoperative local infiltration analgesia of the joint with $300 \mathrm{mg}$ of ropivacaine, $30 \mathrm{mg}$ of ketorolac, and $0.6 \mathrm{mg}$ of epinephrine prior to skin closure. A general anesthesia technique is used for TKA and THA if there are contraindications to use of spinal anesthesia (e.g., history of lumbar spinal surgery, ongoing infection over the lumbar spine, bleeding diathesis, increased intracranial pressure, known allergy to local anesthetics, and patient refusal). All our orthopedic surgeons and anesthesiologists follow this protocol (spinal anesthesia with multimodal analgesia) for THA and TKA and it has been developed through discussion and consensus. Audits of our database have shown the choice of anesthesia technique for lower limb arthroplasties is mostly based on patient (medical condition)-related factors. The standard postoperative active physiotherapy regimen is supervised by physiotherapists twice daily during the in-hospital stay. ${ }^{28}$

Postoperatively, patients receive regular doses of acetaminophen (650-1,000 mg every six hours for five days) and non-steroidal anti-inflammatory drugs (NSAIDs) (celecoxib 100-200 mg twice daily or meloxicam 7.5-15 $\mathrm{mg}$ daily for five days, unless contraindicated). Breakthrough $\mathrm{p}+$ ain is managed with PCOA for all patients following THA or TKA at our hospital unless contraindicated. Patients deemed to be at high risk of opioid diversion (current or past history of substance abuse) or those with preoperative pain NRS $\geq 4 / 10$ despite use of oral analgesics (anti-inflammatory medications and or opioids) are not offered PCOA. All patients undergoing major joint arthroplasties at our hospital attend a preoperative education session that includes information about the surgery, postoperative rehabilitation, and analgesia including PCOA. The PCOA prescription consists of a set dose of a short-acting oral opioid, typically oxycodone or morphine $(5 \mathrm{mg})$ or hydromorphone $(1 \mathrm{mg})$ that is provided at the patient's bedside to be taken at a maximum of two doses every two hours. Patient-controlled oral analgesia use is monitored by the APS team and ward nurses and the medications, if consumed, are replenished by a nurse following a set "lockout" period of two hours. Patients are required to document NRS scores for pain and the time of dosing when they ingest the medication on a PCOA flowsheet. As per protocol, an IV-PCA dispensing hydromorphone or morphine is routinely prescribed as a replacement for PCOA, to be initiated by the ward nurse, in the event of pain not adequately controlled by PCOA (NRS $\geq 4 / 10$ ) after two doses of PCOA within two hours or if the patient is unable to tolerate oral intake because of nausea and vomiting. Nevertheless, an inability to ingest the medications because of nausea and vomiting is uncommon and is usually addressed by switching to a different opioid as advised by the APS. This regimen (antiinflammatory medications and PCOA) is used for over $95 \%$ of the patients undergoing major joint arthroplasties at our hospital. For patients on chronic opioid therapy, longacting opioids are continued postoperatively with dosages often increased by $25-50 \%$ by the APS, while PCOA dosages are also increased by $50-100 \%$. Patients are discharged from APS once stable on oral analgesics, and discharged home usually within $24 \mathrm{hr}$ of end of care by APS with the same oral regimen with the exception of discontinuation of NSAIDs.

\section{Outcome of interest}

The primary outcome for this study was success or failure of PCOA, with failure defined as its termination and initiation of IV-PCA secondary to moderate-to-severe pain (NRS pain score $4 / 10$ or higher) within the first 24 postoperative hours. The decision to replace PCOA with IV-PCA was made by the nurse caring for the patient and or the APS team in the event of pain not being adequately controlled (pain NRS $\geq 4 / 10$ ) after two doses of PCOA within two hours.

\section{Predictor variables of interest}

Our approach to multivariable modelling changed from our initial submission after recommendations from peer reviewers. Therefore, this analysis is primarily exploratory, meaning caution must be applied in appraising its outputs. The primary outcome of this study-success or failure of PCOA-could have been impacted by patient-, anesthetic-, or surgery-related factors.

The association of the outcome with these factors was assessed using univariable analysis and multivariable logistic regression). These variables (factors) were selected based on biologic plausibility and published literature suggesting known or suspected association with higher intensity of pain in the postoperative period following THA or TKA., ${ }^{4,6}$ The clinically relevant predictors of PCOA success or failure that were postulated to be relevant in this study included age, sex, type of arthroplasty (TKA $v s$ THA), preoperative chronic use of controlled-release opioids, history of a chronic pain syndrome, type of anesthetic technique (general $v s$ spinal anesthesia), and perioperative administration of intrathecal morphine. 
Figure Flow diagram indicating choice of postoperative analgesic technique following total hip or knee arthroplasty (TKA or THA)

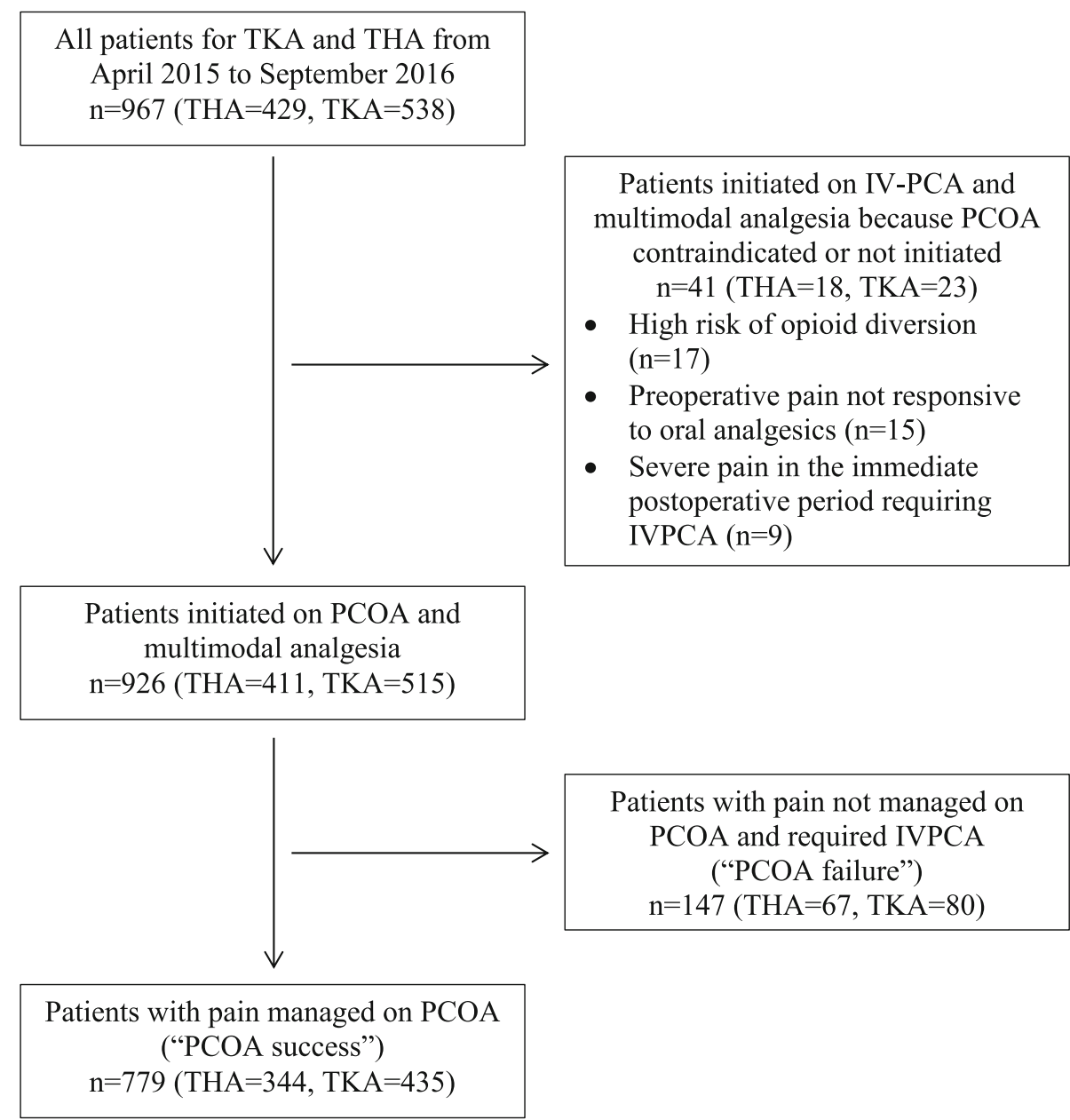

Statistical analysis

The analytic data set was created by a trained data analyst independent from the study team. The analysis compared the aforementioned patient, anesthetic, and surgical characteristics between patients whose postoperative pain was adequately treated with PCOA $v s$ patients with failure of PCOA necessitating use of IV-PCA.

\section{UNIVARIABLE ANALYSIS}

The normality for continuous variables was assessed with the Kolmogorov-Smirnov test. Continuous variables are presented as mean (standard deviation) or median [interquartile range] whereas the categorical variables are summarized by numbers and percentages. Variables with normal distribution were analyzed using $t$ tests, and those with non-normal distribution were analyzed using Wilcoxon rank-sum tests. All $t$ tests were two-sided and unpaired. Categorical variables were analyzed using Chi square test (or Fisher's exact test when any cell had an expected count of less than 5). To compare the variables between PCOA success and failure groups, standardized mean differences with $95 \%$ confidence intervals (CIs) were calculated for continuous variables. Standardized differences were reported as effect sizes for categorical variables (phi coefficient for 2x2 and Cramer's $\mathrm{V}$ for larger tables).

\section{Multivariable LOGISTIC REGRESSION}

The outcome of interest was dichotomous-success or failure of PCOA. The following seven variable were included in the model: age, female sex, type of arthroplasty (THA vs THA), history of chronic use of long-acting (controlled-release) opioids in the preoperative period, history of chronic pain syndrome, administration of intrathecal morphine as part of the anesthetic regimen, and use of general anesthesia ( $v s$ spinal anesthesia). The maximum likelihood estimation method was used to determine the best regression line for the model and the likelihood ratio (LR) omnibus test was used to determine a significant association between the outcome and predictor variables. After the LR omnibus test was found to be 
significant, the Wald test was used to identify the variables that were statistically important. Finally, key assumptions about the model were verified. The details of this process are described in the Electronic Supplementary Material [ESM] eAppendix 1.

Results were reported as odds ratios (OR) with $99 \%$ confidence intervals $(\mathrm{CI})$ and a $P$ value of less than 0.01 was considered significant. All statistical analyses were performed using SAS statistical software version 9.3.3 (SAS Institute, Cary, NC, USA).

\section{Sample size}

We estimated around 90\% of our patients receive spinal anesthesia for THA or TKA. A review of our data as part of quality improvement projects indicated an overall PCOA failure rate of $13 \%$ at our institution. Based on this estimate, a sample size of 251 participants per group, with a significance level of 0.05 , would provide $90 \%$ power to detect a $10 \%$ increase in the incidence of PCOA failure in the general anesthesia group. Nevertheless, we included all available participants during the study period as data were readily available.

\section{Missing data}

Main outcome and exposure variables were available for all participants. We expected missing data for some laboratory test results, but we did not plan to impute data for these gaps because these data were not of direct relevance to our outcome. These data entries with missing data on the laboratory variables accounted for approximately $7 \%$ of the original data set and these were removed from the data set used for analysis. Data on patients with missing values for one or more predictor variables of interest were not excluded. We also accessed anesthetic records of patients who underwent THA or TKA during the study period to establish the anesthetic technique employed for the operation (spinal or general anesthesia) and to populate data on missing variables of interest.

\section{Results}

A total of 926 patients who underwent THA $(n=411)$ or TKA $(n=515)$ during the study period at our hospital and who were initiated on PCOA were identified from our NOPAIn database. Forty-one patients who underwent THA or TKA were not offered PCOA because of the contraindications mentioned in the previous section. Arthroplasties were predominantly unilateral (98.5\% of THA and $96.7 \%$ of TKA). Overall, PCOA failed in 147 patients (15.9\%; 95\% CI, 13.6 to 18.4$)$; of those, $67(45.6 \%)$ underwent THA and $80(54.4 \%)$ TKA. The Figure is a flow diagram indicating choice of postoperative analgesic technique following TKA or THA for the study cohort. A univariable analysis was performed for all demographic, patient, anesthetic, and surgical characteristics for the combined cohort of all THA and TKA patients (Table 1). Subgroup analyses of THA and TKA cohorts were subsequently performed (ESM eTables 1 and 2).

Multivariable logistic regression analysis

The outcome of interest was dichotomous-success or failure of PCOA (the latter characterized by initiation of IV-PCA to treat moderate-to-severe pain). The following seven variable were included in the model: age, sex, type of arthroplasty (THA vs THA), chronic use of controlledrelease opioids in the preoperative period, history of chronic pain syndrome, administration of intrathecal morphine as part of the anesthetic regimen, and use of general anesthesia ( $v s$ spinal anesthesia). The variables associated with an increased probability of PCOA failure included younger age (adjusted OR [aOR] per year of age, $0.97 ; 99 \% \mathrm{CI}, 0.95$ to $0.99 ; P<0.001)$, chronic use of controlled-release opioids (aOR, 3.45; 99\% CI, 1.60 to 7.35; $P<0.001$ ), and use of general anesthesia $v s$ spinal anesthesia (aOR, 2.86; 99\% CI, 1.20 to $6.84 ; P=0.002$ ) (Table 2).

The Hosmer-Lemeshow goodness-of-fit test indicated a well-calibrated model $(P=0.60)$. The concordance (c)statistic (the area under the receiver operating characteristic curve) was 0.71 suggesting a model with moderate discrimination (ESM, eFig. 1). The presence of multicollinearity was checked in the final model. The variance inflation factor was less than 2.5 for each of the predictor variables, hence no collinearity of predictors was identified. The absence of a large number of influential observations was verified by examining influence diagnostics. We found only a few influential observations, and these were not removed (ESM eFig. 2). We also checked to ensure the variable "age" met the assumptions of a linear association (ESM eAppendix 2). We used bootstrapping techniques to confirm internal validity of the prediction model because the parameter estimates were stable (ESM eAppendix 3).

\section{Discussion}

This study showed the feasibility of the administration of PCOA in over 900 patients who underwent THA or TKA. Postoperative pain in the majority of patients initiated on 
Table 1 Characteristics and univariable analysis of all THA and TKA patients with and without PCOA failure

\begin{tabular}{|c|c|c|c|}
\hline Characteristic & $\begin{array}{l}\text { PCOA success } \\
(n=779)\end{array}$ & $\begin{array}{l}\text { PCOA failure } \\
(n=147)\end{array}$ & $\begin{array}{l}\text { Standardized } \\
\text { mean difference }(95 \% \mathrm{CI}) \text { or effect size }\end{array}$ \\
\hline Incidence $(95 \% \mathrm{CI})$ & $84.1 \%(81.6 \%$ to $86.4 \%)$ & $15.9 \%(13.6 \%$ to $18.4 \%)$ & - \\
\hline Age (yr) & $65.2(11.8)$ & $60.1(11.6)$ & $4.25(2.18$ to 6.33$)$ \\
\hline Female sex & $476(61.1 \%)$ & $105(71.4 \%)$ & -0.078 \\
\hline \multicolumn{4}{|l|}{ Procedure type } \\
\hline Unilateral TKA & $422(54.2 \%)$ & $76(51.2 \%)$ & 0.032 \\
\hline Unilateral THA & $339(43.5 \%)$ & $66(44.9 \%)$ & \\
\hline Bilateral TKA & $13(1.7 \%)$ & $4(2.7 \%)$ & \\
\hline Bilateral THA & $5(0.6 \%)$ & $1(0.68 \%)$ & \\
\hline Primary procedure & $696(89.4 \%)$ & $134(91.2 \%)$ & -0.022 \\
\hline \multicolumn{4}{|l|}{ Comorbidities } \\
\hline HTN or CAD & $373(47.9 \%)$ & $76(51.7 \%)$ & 0.028 \\
\hline Smoker or ex-smoker or COPD & $135(17.3 \%)$ & $36(24.5 \%)$ & 0.067 \\
\hline Anxiety or depression & $6(0.77 \%)$ & $2(1.36 \%)$ & 0.023 \\
\hline OSA & $112(14.4 \%)$ & $22(15.0 \%)$ & 0.006 \\
\hline Obesity & $61(7.8 \%)$ & $18(12.2 \%)$ & 0.058 \\
\hline Asthma & $54(6.9 \%)$ & $11(7.5 \%)$ & 0.008 \\
\hline Diabetes & $103(13.2 \%)$ & $18(12.2 \%)$ & -0.011 \\
\hline Any chronic pain syndrome & $17(2.2 \%)$ & $9(6.1 \%)$ & 0.087 \\
\hline Renal disease & $28(3.6 \%)$ & $6(4.1 \%)$ & 0.009 \\
\hline Neuropathy & $8(1.0 \%)$ & $2(2.0 \%)$ & 0.034 \\
\hline Cancer & $10(1.3 \%)$ & $3(2.0 \%)$ & 0.024 \\
\hline Substance dependency & $13(1.7 \%)$ & $5(3.4 \%)$ & 0.046 \\
\hline \multicolumn{4}{|l|}{ Preoperative medications: } \\
\hline Short-acting opioids & $140(18.0 \%)$ & $47(32.0 \%)$ & 0.127 \\
\hline Long-acting opioids & $38(4.9 \%)$ & $23(15.7 \%)$ & 0.159 \\
\hline NSAIDs/acetaminophen & $313(40.2 \%)$ & $66(44.9 \%)$ & 0.035 \\
\hline Antidepressants & $69(8.9 \%)$ & $24(16.3 \%)$ & 0.091 \\
\hline Statins & $190(24.4 \%)$ & $27(18.4 \%)$ & -0.052 \\
\hline Anticonvulsants & $54(6.9 \%)$ & $19(12.9 \%)$ & 0.081 \\
\hline Sedatives/BZDs & $68(8.7 \%)$ & $15(10.2 \%)$ & 0.019 \\
\hline Steroids & $11(1.4 \%)$ & $5(3.4 \%)$ & 0.056 \\
\hline Cannabinoids & $14(1.8 \%)$ & $7(4.8 \%)$ & 0.073 \\
\hline Muscle relaxants/ antispasmodics & $4(0.5 \%)$ & $2(1.4 \%)$ & 0.039 \\
\hline Any neuropathic pain medication & $103(13.2 \%)$ & $37(15.2 \%)$ & 0.122 \\
\hline Spinal anesthesia & $725(93.2 \%)$ & $117(79.6 \%)$ & -0.174 \\
\hline Intrathecal morphine & $615(79.0 \%)$ & $95(64.6 \%)$ & -0.124 \\
\hline ACB (TKA patients) & $180(41.4 \%)$ & $37(55.1 \%)$ & 0.037 \\
\hline Preoperative creatinine $\left(\mathrm{mmol} \cdot \mathrm{L}^{-1}\right)$ & $79.1(38.0)$ & $79.4(34.5)$ & $-0.33(-11.5$ to 10.84$)$ \\
\hline NRS on movement at $24 \mathrm{hr}$ after surgery & $4.4(3.6)$ & $5.6(3.8)$ & $-1.19(-1.83$ to -0.56$)$ \\
\hline \multicolumn{4}{|l|}{ Prescribed APS medications } \\
\hline Acetaminophen & $757(97.2 \%)$ & $96.60 \%$ & -0.013 \\
\hline NSAIDs & $578(4.2 \%)$ & $107(72.8 \%)$ & -0.012 \\
\hline
\end{tabular}


Table 1 continued

\begin{tabular}{llll}
\hline Characteristic & $\begin{array}{l}\text { PCOA success } \\
(n=779)\end{array}$ & $\begin{array}{l}\text { PCOA failure } \\
(n=147)\end{array}$ & $\begin{array}{l}\text { Standardized } \\
\text { mean difference (95\% CI) or effect size }\end{array}$ \\
\hline CR opioid* & $27(3.5 \%)$ & $17(11.6 \%)$ & 0.139 \\
\hline
\end{tabular}

Results are presented as mean (standard deviation) or $n(\%)$

$\mathrm{ACB}=$ adductor canal block; $\mathrm{APS}=$ acute pain service; $\mathrm{CAD}=$ coronary artery disease $\mathrm{COPD}=$ chronic obstructive pulmonary disease; $\mathrm{CR}=$ controlled release; HTN = hypertension; NSAIDs = non-steroidal anti-inflammatory drugs; OSA = obstructive sleep apnea; PCOA = patientcontrolled oral analgesia; THA = total hip arthroplasty; TKA = total knee arthroplasty

* Not every patient on long-acting opioids prior to surgery receives these medications in the postoperative period. Patients who are drowsy in the postoperative recovery room may have long-acting opioids reduced or discontinued because of concerns about exacerbating the drowsiness and compromising their respiratory status

\# Standardized mean difference with $95 \%$ confidence intervals (CI) were calculated for continuous variables. Standardized differences were reported as effect sizes for categorical variables (phi coefficient for $2 \times 2$ and Cramer's $\mathrm{V}$ for larger tables)

Table 2 Multivariable regression analysis of risk factors associated with failure of patient-controlled oral analgesia in patients undergoing total knee and total hip arthroplasty

\begin{tabular}{|c|c|c|c|c|c|c|}
\hline \multirow[b]{2}{*}{ Characteristic } & \multicolumn{3}{|l|}{ Unadjusted } & \multicolumn{3}{|l|}{ Adjusted } \\
\hline & Odds ratio & $99 \% \mathrm{CI}$ & $P$ value & Odds ratio & $99 \% \mathrm{CI}$ & $P$ value \\
\hline Age $\dagger$ & 0.97 & 0.96 to 0.99 & $<0.0001$ & 0.97 & 0.95 to 0.99 & $<0.001$ \\
\hline Female sex & 1.59 & 0.96 to 2.64 & 0.012 & 1.67 & 0.97 to 2.86 & 0.01 \\
\hline TKA vs THA & 0.94 & 0.59 to 1.50 & 0.75 & 0.83 & 0.51 to 1.36 & 0.33 \\
\hline Preoperative use of controlled-release opioids & 3.57 & 1.75 to 7.46 & $<0.001$ & 3.45 & 1.60 to 7.35 & $<0.001$ \\
\hline History of a chronic pain syndrome & 2.94 & 0.98 to 8.70 & 0.01 & 2.44 & 0.78 to 7.69 & 0.04 \\
\hline Lack of intrathecal morphine & 2.05 & 1.25 to 3.38 & $<0.001$ & 1.40 & 0.70 to 2.81 & 0.22 \\
\hline General anesthesia vs spinal anesthesia & 3.51 & 1.85 to 6.68 & $<0.001$ & 2.86 & 1.20 to 6.84 & 0.002 \\
\hline
\end{tabular}

$\mathrm{CI}=$ confidence interval; THA = total hip arthroplasty; TKA = total knee arthroplasty

$\dagger$ Unit year increase in age

PCOA was successfully managed with this modality (84.1\%; 95\% CI, 81.6 to 86.4). Younger age, preoperative chronic use of controlled-release opioids, and use of general anesthesia was associated with failure of PCOA associated with moderate-to-severe pain.

Data source and importance of our outcomes

This is the first study of data extracted from a large and reliably populated clinical database (NOPAIn) of perioperative analgesic course following lower limb arthroplasties. Though some other studies have attempted to identify factors associated with moderate-to-severe pain in the postoperative period, ${ }^{4}$ these attempts have mostly relied on administrative healthcare and or billing databases that are limited by lack of clinicians' input and missing data. A unique feature of our database is the requirement for populating it in real-time during delivery of clinical care. The data from this database that were used for this study were cross-referenced against electronic health records for demographics, type of anesthesia, use of nerve blocks, comorbidities, prescription medications, and serum creatinine values. Furthermore, the database used in this study involved use of raw data for analysis with mandatory data entry at point-of-care reducing the potential for missing and erroneous data.

Therapeutic success with PCOA in the first 24 postoperative hours is an important outcome because its failure reflects poorly treated pain in the initial 24 postoperative hours. Moderate-to-severe pain in the initial postoperative period following THA and TKA can be associated with delayed ambulation, ${ }^{29}$ increased length of stay in the hospital, higher costs, ${ }^{5,6,30}$ more complications including thromboembolic and infectious events, ${ }^{29}$ and persistent postoperative pain. $^{8}$ The failure of PCOA indicates moderate-to-severe pain but the incidence of this adverse outcome was considerably lower in our study (15.9\%; 95\% CI, 13.6 to 18.4) than previously reported figures $(20-58 \%))^{4,31-33}$ The findings of our study highlight the feasibility of routinely co-administering PCOA with a 
comprehensive multimodal analgesic regimen inclusive of regional anesthesia for patients undergoing THA and TKAoperations that can be associated with significant pain in the postoperative period. ${ }^{4,34}$ The interventions in our multimodal analgesic regime are inexpensive, universally available, and easy to implement. The utility of PCOA is further shown by the high prevalence of risk factors for moderate-to-severe postoperative pain such as chronic pain, preoperative opioid use, depression, and anxiety disorders ${ }^{35-38}$ in our study cohort. Using PCOA can avoid the need for IV-PCA, allowing for lower healthcare costs, reduced associated complication rates, ${ }^{12,13}$ and enhanced patient mobility. Furthermore, compared with nurse-administered oral opioids, PCOA maintains the ability to provide adequate timely postoperative analgesia with the benefits of patient autonomy, easy use, and improved pain control. ${ }^{39,40}$

\section{Factors predictive for PCOA failure}

Our study identified a number of factors associated with PCOA failure. Though univariable analysis of the cohort identified several factors, multivariable logistic regression analysis included seven variables (age, sex, TKA vs THA, history of a chronic pain syndrome, preoperative use of controlled-release opioids, use of intrathecal morphine, and general vs spinal anesthesia). Younger age was associated with PCOA failure in our study. This is in keeping with previous studies showing an inverse relationship between age and moderate-to-severe pain following arthroplasty. ${ }^{4}$ Nevertheless, sex of the patient was not found to be a significant factor for the whole cohort in this study. Female sex is often reported to be associated with more severe and persistent pain after lower limb arthroplasty ${ }^{4,41,42}$ and other operations. ${ }^{43-45}$ While sex differences in pain responses are multifactorial, ${ }^{46}$ differences in pain severity specific to arthroplasty have previously been attributed to women having greater disability at the time of surgery. ${ }^{47,48}$ Other trials have reported inconsistent ${ }^{51}$ differences in pain scores ${ }^{49-52}$ and analgesic consumption in women undergoing TKA. $^{49}$ Preoperative chronic use of controlled-release opioids was also associated with PCOA failure despite the APS team pre-emptively increasing the dose of sustained- and immediate-release opioids. Chronic pain and use of controlled-release opioids are common preoperatively in this population ${ }^{53}$ and both these variables are associated with severe postoperative pain following arthroplasty. ${ }^{4,54,55}$ Lastly, use of general ( $v s$ spinal) anesthesia was also associated with PCOA failure in our study cohort. Published literature also reflects this finding with neuraxial anesthesia associated with better patient outcomes ${ }^{56,57}$ and it is recommended for fast-track arthroplasty. ${ }^{58}$
Lower oral opioid consumption was also associated with PCOA failure in our study, suggesting that oral opioid dosages were inadequate for a subset of patients, necessitating conversion to IV-PCA early in the postoperative course. Such patients likely experienced a greater severity of postoperative pain $^{44}$ that was either inadequately treated with PCOA or required early interventions in the form of PCOA dose escalation. ${ }^{41}$ This effect was likewise found in the THA and TKA cohorts, keeping with previous evidence of correlation of postoperative opioid consumption with poorly controlled acute pain following THA $^{59}$ and TKA. ${ }^{39}$ Failure rates of PCOA can be reduced by ensuring it is offered to patients who are able to understand its role and use it effectively. Preoperative education in use of PCOA may help identify patients who may not be appropriate candidates for this modality. Early identification of patients at risk of PCOA failure would inform the prescription of higher doses of oral opioids or substitution with intravenous analgesics and multimodal analgesia to match their higher postoperative requirements.

It should be recognized that PCOA has several advantages, but its delivery is subject to specific factors, including bioavailability, that affect pharmacokinetics of opioids administered orally. Patients who have sedation, confusion, or delirium are also likely to be at risk for inadequate use of regimens involving oral self-administration of opioids ${ }^{60}$ and these patients are often prescribed other forms of analgesia for breakthrough pain (e.g., nurse-administered analgesia). Another issue to be aware of is that patients with a history of opioid abuse are excluded from PCOA administration because of risk of diversion.

Our analysis did not show an association between other variables previously shown to be associated with moderateto-severe post-arthroplasty pain such as arthroplasty type (TKA being associated with more severe pain than THA), chronic pain, anxiety, and depression. ${ }^{42}$ The lack of association between a diagnosis of anxiety and or depression with poorer analgesic experience in our study may reflect lack of data on current levels of these psychological comorbidities in our cohort.

While the overall PCOA success rate at our hospital for patients initiated on this modality supports the analgesic efficacy of this regimen, factors predictive of failure identified in our study can help in formulating analgesic strategies for patients who may benefit from an individualized approach to acute pain management. Furthermore, in accepting PCOA failure as a surrogate marker for moderate-to-severe pain, the incidence of pain of this intensity was lower in our cohort than the 20-58\% reported in other studies. ${ }^{4,31-33}$ Based on these findings, we propose that a multimodal analgesic regimen incorporating 
PCOA is a viable analgesic strategy for most patients following lower limb arthroplasty.

\section{Generalizability of results}

Significant institutional variability exists in perioperative anesthetic and postoperative analgesic regimens for arthroplasty, ${ }^{56,58,61}$ with general anesthesia being the most common anesthetic modality. ${ }^{62,63}$ Therefore, the administration of PCOA may be associated with differing outcomes in other centres. Our centre uses guidelinerecommended multimodal analgesia regimens ${ }^{64,65}$ that play a role in keeping PCOA failure rates low. Use of PCOA may thus be particularly effective for total joint arthroplasty in centres where these guidelines are also applied. Our study also suggests the possibility of better analgesic outcomes in the early postoperative period when spinal anesthesia is used for lower limb arthroplasty. These findings should encourage other centres to consider using neuraxial anesthesia for major joint arthroplasties in the lower limbs. Further, we acknowledge clinical practice is currently variable with regards to ACB use for TKA but, because of concerns associated with femoral nerve blockade and motor weakness interfering with mobilization, ACB use is increasing. ${ }^{66}$

\section{Limitations}

Our study has a few limitations. The data collection was limited to the first three days of the postoperative period. Nevertheless, our data showed that the majority of patients are discharged from APS within 24 to $48 \mathrm{hr}$ in the postoperative period because this represents the period of most severe pain. ${ }^{9}$ Second, data are lacking on the administration of opioids for patients undergoing general anesthesia, potentially underestimating their 24-hr opioid consumption. Our in-house database does not record pain levels beyond discharge from the hospital. It would have been informative to study the correlation between PCOA failure in the first two postoperative days and persistent postoperative pain. Data are also lacking on certain additional variables associated with moderate-to-severe pain following arthroplasty, namely body mass index, preoperative (baseline) pain levels, previous surgery at surgical site, pain at other sites (e.g., back pain), ${ }^{35,67}$ and certain predictive psychological variables associated with pain (e.g., catastrophizing). ${ }^{51}$ Nevertheless, the lack of data on preoperative pain levels is in part offset by data on preoperative chronic opioid use, which often correlate with the presence of preoperative moderate-to-severe pain. We also acknowledge an important deficiency of our NOPAIn database-a lack of data on the amount of opioid consumed by patients through PCOA and IV-PCA in the postoperative period. Furthermore, we did not analyze the incidence of potential adverse effects of PCOA such as nausea, vomiting, sedation, and respiratory depression.

This study is based on the experience of a single centre and there may be challenges to generalizability of its results to centres that have different perioperative analgesic protocols. Our prediction model involved selection of variables based on biologic plausibility and literature. This approach may have resulted in a failure to examine all relevant variables. The primary outcome (success or failure of PCOA) may have been impacted by factors such as inadequate dosage and there is also a risk of misclassification bias. There are also valid concerns about unsupervised consumption of opioids (as occurs with PCOA) increasing the likelihood of diversion. Healthcare providers must remain vigilant about the possibility of diversion of opioids given to patients for unsupervised consumption as part of PCOA.

\section{Conclusions}

Our study showed that routine administration of PCOA as part of a multimodal analgesic strategy is feasible following THA and TKA with a relatively low incidence of PCOA failure. A number of risk factors for PCOA failure were identified, including younger age, preoperative chronic use of controlled-release opioids, and use of general anesthesia. These characteristics may be used to supplement clinical judgement in deciding whether to prescribe PCOA or IV-PCA as the primary breakthrough analgesic modality following THA or TKA.

Author contributions Leon Vorobeichik contributed to data acquisition, analysis and interpretation, and writing the manuscript. Yasmine Hoydonckx and Pranab Kumar contributed to data analysis and interpretation, and writing the manuscript. Arlene Buzon-Tan and Susan Walker contributed to data acquisition and interpretation, and writing the manuscript. Kyle Kirkham, Lashmi Venkatraghavan, and Atul J. Prabhu contributed to interpretation of data analysis and writing the manuscript. Dharini Ilangomaran contributed to design of the NOPAIn database and data acquisition. Anuj Bhatia contributed to conception of the study idea and design, acquisition, analysis and interpretation of data, and writing the manuscript.

Acknowledgements The authors would like to thank Alex Kiss, $\mathrm{PhD}$, Department of Research Design and Biostatistics, Sunnybrook Health Sciences Centre, Institute for Clinical Evaluative Sciences, University of Toronto for his help with the statistical analysis.

\section{Disclosures None.}

Funding statement This work was supported by departmental funding from Department of Anesthesia and Pain Management at University Health Network-Toronto Western Hospital. 
Editorial responsibility This submission was handled by Dr. Gregory L. Bryson, Deputy Editor-in-Chief, Canadian Journal of Anesthesia.

\section{References}

1. Patel A, Pavlou G, Mújica-Mota RE, Toms AD. The epidemiology of revision total knee and hip arthroplasty in England and Wales: a comparative analysis with projections for the United States. A study using the National Joint Registry dataset. Bone Joint J 2015; 97-B: 1076-81.

2. Räsänen P, Paavolainen $P$, Sintonen $H$, et al. Effectiveness of hip or knee replacement surgery in terms of quality-adjusted life years and costs. Acta Orthop 2007; 78: 108-15.

3. Lizaur-Utrilla A, Martinez-Mendez D, Miralles-Muñoz FA, Marco-Gomez L, Lopez-Prats FA. Risk-benefit on quality of life after total knee arthroplasty in octogenarians. J Arthroplasty 2017; 32: 2417-20.

4. Liu SS, Buvanendran A, Rathmell JP, et al. Predictors for moderate to severe acute postoperative pain after total hip and knee replacement. Int Orthop 2012; 36: 2261-7.

5. Russo $M W$, Parks NL, Hamilton WG. Perioperative pain management and anesthesia: a critical component to rapid recovery total joint arthroplasty. Orthop Clin North Am 2017; 48: 401-5.

6. Aasvang EK, Luna IE, Kehlet $H$. Challenges in postdischarge function and recovery: the case of fast-track hip and knee arthroplasty. Br J Anaesth 2015; 115: 861-6.

7. Blågestad T, Pallesen S, Grønli J, Tang NK, Nordhus IH. How perceived pain influence sleep and mood more than the reverse: a novel, exploratory study with patients awaiting total hip arthroplasty. Front Psychol 2016; DOI: https://doi.org/10.3389/ fpsyg.2016.01689.

8. Puolakka PA, Rorarius MG, Roviola M, Puolakka TJ, Nordhausen $K$, Lindgren $L$. Persistent pain following knee arthroplasty. Eur J Anaesthesiol 2010; 27: 455-60.

9. $L i J W, M a Y S$, Xiao $L K$. Postoperative pain management in total knee arthroplasty. Orthop Surg 2019; 11: 755-61.

10. Mordin M, Anastassopoulos K, van Breda A, et al. Clinical staff resource use with intravenous patient-controlled analgesia in acute postoperative pain management: results from a multicenter, prospective, observational study. J Perianesth Nurs 2007; 22: 243-55.

11. Choinière $M$, Rittenhouse BE, Perreault $S$, et al. Efficacy and costs of patient-controlled analgesia versus regularly administered intramuscular opioid therapy. Anesthesiology 1998; 89: 1377-88.

12. Palmer P, Ji X, Stephens J. Cost of opioid intravenous patientcontrolled analgesia: results from a hospital database analysis and literature assessment. Clinicoecon Outcomes Res 2014; 6: 311-8.

13. Jeleazcov C, Ihmsen H, Saari TI, et al. Patient-controlled analgesia with target-controlled infusion of hydromorphone in postoperative pain therapy. Anesthesiology 2016; 124: 56-68.

14. Schein JR, Hicks RW, Nelson WW, Sikirica V, Doyle DJ. Patientcontrolled analgesia-related medication errors in the postoperative period: causes and prevention. Drug Saf 2009; 32: 549-59.

15. Lamplot JD, Wagner ER, Manning $D W$. Multimodal pain management in total knee arthroplasty: a prospective randomized controlled trial. J Arthroplasty 2014; 29: 329-34.

16. Bonnal A, Dehon A, Nagot N, Macioce V, Nogue E, Morau E. Patient-controlled oral analgesia versus nurse-controlled parenteral analgesia after caesarean section: a randomised controlled trial. Anaesthesia 2016; 71: 535-43.

17. Rothwell MP, Pearson D, Hunter JD, et al. Oral oxycodone offers equivalent analgesia to intravenous patient-controlled analgesia after total hip replacement: a randomized, singlecentre, non-blinded, non-inferiority study. Br J Anaesth 2011; 106: 865-72.

18. Kastanias P, Gowans S, Tumber PS, Snaith K, Robinson S. Patient-controlled oral analgesia for postoperative pain management following total knee replacement. Pain Res Manag 2010; 15: 11-6.

19. Rothwell MP, Pearson D, Hunter JD, et al. Constipation after total hip replacement may be lower with oral opioid analgesia than with intravenous opioid analgesia. Br J Anaesth 2012; 108: 528-9.

20. Donell S, Deane $K$, Swift $L$, et al. Patient directed self management of pain (PaDSMaP) compared to treatment as usual following total knee replacement: study protocol for a randomized controlled trial. Trials 2012; DOI: https://doi.org/10. 1186/1745-6215-13-204.

21. Kastanias $P$, Snaith KE, Robinson S. Patient-controlled oral analgesia: a low-tech solution in a high-tech world. Pain Manag Nurs 2006; 7: 126-32.

22. Striebel HW, Römer M, Kopf A, Schwagmeier R. Patient controlled oral analgesia with morphine. Can J Anaesth 1996; 43: 749-53.

23. Striebel HW, Scheitza W, Philippi W, Behrens U, Toussaint S. Quantifying oral analgesic consumption using a novel method and comparison with patient-controlled intravenous analgesic consumption. Anesth Analg 1998; 86: 1051-3.

24. Brummett CM, Clauw DJ. Flipping the paradigm: from surgeryspecific to patient-driven perioperative analgesic algorithms. Anesthesiology 2015; 122: 731-3.

25. von Elm E, Altman DG, Egger $M$, et al. Strengthening the Reporting of Observational Studies in Epidemiology (STROBE) statement: guidelines for reporting observational studies. BMJ 2007; 335: 806-8

26. Benchimol EI, Smeeth L, Guttmann A, et al. The REporting of studies Conducted using Observational Routinely-collected health Data (RECORD) statement. PLoS Med 2015; DOI: https://doi.org/10.1371/journal.pmed.1001885.

27. Jovaisas B. Opioids. CPS 2016: Compendium of pharmaceuticals and specialties. Canadian Pharmacists Association 2016: 2334.

28. Perlas A, Chan VW, Beattie S. Anesthesia technique and mortality after total hip or knee arthroplasty: a retrospective, propensity score-matched cohort study. Anesthesiology 2016; 125: 724-31

29. Pearse EO, Caldwell BF, Lockwood RJ, Hollard J. Early mobilisation after conventional knee replacement may reduce the risk of postoperative venous thromboembolism. J Bone Joint Surg Br 2007; 89: 316-22.

30. Kehlet H. Fast-track hip and knee arthroplasty. Lancet $2013 ; 381$ : $1600-2$.

31. Pinto PR, McIntyre T, Ferrero $R$, Almeida A, Araújo-Soares $V$. Risk factors for moderate and severe persistent pain in patients undergoing total knee and hip arthroplasty: a prospective predictive study. PLoS One 2013; DOI: https://doi.org/10.1371/ journal.pone.0073917.

32. Chan EY, Blyth FM, Nairn L, Fransen M. Acute postoperative pain following hospital discharge after total knee arthroplasty. Osteoarthritis Cartilage 2013; 21: 1257-63.

33. Wylde V, Rooker J, Halliday L, Blom A. Acute postoperative pain at rest after hip and knee arthroplasty: severity, sensory qualities and impact on sleep. Orthop Traumatol Surg Res 2011; 97: 139-44.

34. Beswick AD, Wylde V, Gooberman-Hill R, Blom A, Dieppe P. What proportion of patients report long-term pain after total hip 
or knee replacement for osteoarthritis? A systematic review of prospective studies in unselected patients. BMJ Open 2012; DOI: https://doi.org/10.1136/bmjopen-2011-000435.

35. Lewis GN, Rice DA, McNair PJ, Kluger M. Predictors of persistent pain after total knee arthroplasty: a systematic review and meta-analysis. Br J Anaesth 2015; 114: 551-61.

36. Jorgensen CC, Knop J, Nordentoft M, Kehlet H; Lundbeck Foundation Centre for Fast-track Hip and Knee Replacement Collaborative Group. Psychiatric disorders and psychopharmacologic treatment as risk factors in elective fasttrack total hip and knee arthroplasty. Anesthesiology 2015; 123 : 1281-91.

37. Rakel BA, Blodgett NP, Zimmerman MB, et al. Predictors of postoperative movement and resting pain following total knee replacement. Pain 2012; 153: 2192-203.

38. Goesling J, Moser SE, Zaidi B, et al. Trends and predictors of opioid use after total knee and total hip arthroplasty. Pain 2016; 157: 1259-65.

39. Lindberg MF, Miaskowski C, Rustøen T, et al. The impact of demographic, clinical, symptom and psychological characteristics on the trajectories of acute postoperative pain after total knee arthroplasty. Pain Med 2017; 18: 124-39.

40. McNicol ED, Ferguson MC, Hudcova J. Patient controlled opioid analgesia versus non-patient controlled opioid analgesia for postoperative pain. Cochrane Database Syst Rev 2015; DOI: https://doi.org/10.1002/14651858.CD003348.pub3.

41. Hernández C, Díaz-Heredia J, Berraquero ML, Crespo P, Loza E, Ruiz Ibán MÁ. Pre-operative predictive factors of postoperative pain in patients with hip or knee arthroplasty: a systematic review. Reumatol Clin 2015; 11: 361-80.

42. Petrovic NM, Milovanovic DR, Ignjatovic Ristic D, Riznic N, Ristic B, Stepanovic Z. Factors associated with severe postoperative pain in patients with total hip arthroplasty. Acta Orthop Traumatol Turc 2014; 48: 615-22.

43. Kehlet H, Jensen TS, Woolf CJ. Persistent postsurgical pain: risk factors and prevention. Lancet 2006; 367: 1618-25.

44. Ip HY, Abrishami A, Peng PW, Wong J, Chung F. Predictors of postoperative pain and analgesic consumption: a qualitative systematic review. Anesthesiology 2009; 111: 657-77.

45. Yen CR, Tsou MY, Mandell MS, et al. An analysis of patient variables that influence intravenous patient-controlled analgesic use of morphine with quantile regression. Anesthesiology 2010; 112: 688-95.

46. Fillingim RB, King CD, Ribeiro-Dasilva MC, Rahim-Williams B, Riley $J L$ Srd. Sex, gender, and pain: a review of recent clinical and experimental findings. J Pain 2009; 10: 447-85.

47. Holtzman J, Saleh $K$, Kane R. Gender differences in functional status and pain in a Medicare population undergoing elective total hip arthroplasty. Med Care 2002; 40: 461-70.

48. Kennedy DM, Hanna SE, Stratford PW, Wessel J, Gollish JD. Preoperative function and gender predict pattern of functional recovery after hip and knee arthroplasty. J Arthroplasty 2006; 21 : 559-66.

49. Pope D, El-Othmani MM, Manning BT, Sepula M, Markwell SJ, Saleh KJ. Impact of age, gender and anesthesia modality on postoperative pain in total knee arthroplasty patients. Iowa Orthop J 2015; 35: 92-8.

50. Kornilov N, Lindberg MF, Gay C, et al. Factors related to postoperative pain trajectories following total knee arthroplasty: a longitudinal study of patients admitted to a Russian orthopaedic clinic. Pain Res Treat 2016; DOI: https://doi.org/10.1155/2016/ 3710312.

51. Roth ML, Tripp DA, Harrison MH, Sullivan M, Carson P. Demographic and psychosocial predictors of acute perioperative pain for total knee arthroplasty. Pain Res Manag 2007; 12: 18594.
52. Barrington JW, Lovald ST, Ong KL, Watson HN, Emerson RH Jr. Postoperative pain after primary total knee arthroplasty: comparison of local injection analgesic cocktails and the role of demographic and surgical factors. J Arthroplasty 2016; 31: 288-92.

53. Hansen CA, Inacio MC, Pratt NL, Roughead EE, Graves SE. Chronic use of opioids before and after total knee arthroplasty: a retrospective cohort study. J Arthroplasty 2017; 32(811-7): e1.

54. Zywiel MG, Stroh DA, Lee SY, Bonutti PM, Mont MA. Chronic opioid use prior to total knee arthroplasty. J Bone Joint Surg Am 2011; 93: 1988-93.

55. Rozell JC, Courtney PM, Dattilo JR, Wu CH, Lee GC. Preoperative opiate use independently predicts narcotic consumption and complications after total joint arthroplasty. J Arthroplasty 2017; 32: 2658-62.

56. Wong PB, McVicar J, Nelligan K, Bleackley JC, McCartney CJ. Factors influencing the choice of anesthetic technique for primary hip and knee arthroplasty. Pain Manag 2016; 6: 297-311.

57. Mauermann WJ, Shilling AM, Zuo Z. A comparison of neuraxial block versus general anesthesia for elective total hip replacement: a meta-analysis. Anesth Analg 2006; 103: 1018-25.

58. Soffin EM, YaDeau JT. Enhanced recovery after surgery for primary hip and knee arthroplasty: a review of the evidence. Br J Anaesth 2016; 117(suppl 3): iii62-72.

59. Pagé MG, Katz J, Curtis K, Lutzky-Cohen N, Escobar EM, Clarke $H A$. Acute pain trajectories and the persistence of post-surgical pain: a longitudinal study after total hip arthroplasty. J Anesth 2016; 30: 568-77.

60. Leung JM, Sands LP, Paul S, Joseph T, Kinjo S, Tsai T. Does postoperative delirium limit the use of patient-controlled analgesia in older surgical patients? Anesthesiology 2009; 111: 625-31.

61. Grosu I, Lavand'homme P, Thienpont E. Pain after knee arthroplasty: an unresolved issue. Knee Surg Sports Traumatol Arthrosc 2014; 22: 1744-58.

62. Fleischut PM, Eskreis-Winkler JM, Gaber-Baylis LK, et al. Variability in anesthetic care for total knee arthroplasty: an analysis from the anesthesia quality institute. Am J Med Qual 2015; 30: 172-9.

63. Gabriel RA, Kaye AD, Jones MR, Dutton RP, Urman RD. Practice variations in anesthetic care and its effect on clinical outcomes for primary total hip arthroplasties. J Arthroplasty 2016; 31: 918-22.

64. Chou R, Gordon DB, de Leon-Casasola OA, et al. Management of postoperative pain: a clinical practice guideline from the American Pain Society, the American Society of Regional Anesthesia and Pain Medicine, and the American Society of Anesthesiologists' Committee on Regional Anesthesia, Executive Committee, and Administrative Council. J Pain 2016; 17: 131-57.

65. Kopp SL, Børglum J, Buvanendran A, et al. Anesthesia and analgesia practice pathway options for total knee arthroplasty: an evidence-based review by the American and European Societies of Regional Anesthesia and Pain Medicine. Reg Anesth Pain Med 2017; 42: 683-97.

66. Masaracchia MM, Herrick MD, Barrington MJ, Hartmann PR, Sites $B D$. Adductor canal blocks: changing practice patterns and associated quality profile. Acta Anaesthesiol Scand 2017; 61: 224-31.

67. Inacio MC, Hansen C, Pratt NL, Graves SE, Roughead EE. Risk factors for persistent and new chronic opioid use in patients undergoing total hip arthroplasty: a retrospective cohort study. BMJ Open 2016; DOI: https://doi.org/10.1136/bmjopen-2015010664.

Publisher's Note Springer Nature remains neutral with regard to jurisdictional claims in published maps and institutional affiliations. 